Purdue University

Purdue e-Pubs

CTRC Research Publications

Cooling Technologies Research Center

2009

Experimental Study of Aerodynamic Damping in Arrays of Vibrating Cantilevers

\author{
M. L. Kimber \\ Purdue University - Main Campus \\ R. Lonergan \\ Purdue University - Main Campus \\ S V. Garimella \\ Purdue University, sureshg@purdue.edu
}

Follow this and additional works at: http:/ / docs.lib.purdue.edu/coolingpubs

Kimber, M. L.; Lonergan, R.; and Garimella, S V., "Experimental Study of Aerodynamic Damping in Arrays of Vibrating Cantilevers" (2009). CTRC Research Publications. Paper 122.

http://dx.doi.org/10.1016/j.jfluidstructs.2009.07.003

This document has been made available through Purdue e-Pubs, a service of the Purdue University Libraries. Please contact epubs@purdue.edu for additional information. 


\title{
Experimental Study of Aerodynamic Damping in Arrays of Vibrating Cantilevers ${ }^{1}$
}

\author{
Mark Kimber², Ronan Lonergan, and Suresh V. Garimella \\ Cooling Technologies Research Center \\ School of Mechanical Engineering and Birck Nanotechnology Center, Purdue University \\ 585 Purdue Mall, West Lafayette, IN 47907-2088 USA
}

\begin{abstract}
Cantilever structures vibrating in a fluid are encountered in numerous engineering applications. The aerodynamic loading from a fluid can have a large effect on both the resonance frequency and damping, and has been the subject of numerous studies. The aerodynamic loading on a single beam is altered when multiple beams are configured in an array. In such situations, neighboring beams interact through the fluid and their dynamic behavior is modified. In this work, aerodynamic interactions between neighboring cantilever beams operating near their first resonance mode and vibrating at amplitudes comparable to their widths are experimentally explored. The degree to which two beams become coupled through the fluid is found to be sensitive to vibration amplitude and proximity of neighboring components in the array. The cantilever beams considered are slender piezoelectric fans (approximately $6 \mathrm{~cm}$ in length), and are caused to vibrate in-phase and out-of-phase at frequencies near their fundamental resonance values. Aerodynamic damping is expressed in terms of the quality factor for two different array configurations and estimated for both in-phase and out-of-phase conditions. The two array configurations considered are for neighboring fans placed face-to-face and edge-to-edge. It is found that the damping is greatly influenced by proximity of neighboring fans and phase difference. For the face-to-face configuration, a reduction in damping is observed for in-phase vibration, while it is greatly increased for out-of-phase vibration; the opposite effect is seen for the edge-to-edge configuration. The resonance frequencies also show a dependence on the phase difference, but these changes are small compared to those observed for damping. Correlations are developed based on the experimental data which can be used to predict the aerodynamic damping in arrays of vibrating cantilevers. The distance at which the beams no longer interact is quantified for both array configurations. Understanding the fluid interactions between neighboring vibrating beams is essential for predicting the dynamic behavior of such arrays and designing them for practical applications.
\end{abstract}

Keywords: fluid coupling, aerodynamic damping, piezoelectric fans, vibrating cantilevers, flow-structure interaction

\footnotetext{
${ }^{1}$ Submitted for possible publication in Journal of Fluids and Structures, October 2008

${ }^{2}$ Author to whom correspondence should be addressed: 412-624-8111,mlk53@pitt.edu
} 


\section{Introduction}

A cantilever vibrating in a fluid is encountered in numerous engineering applications. In the case of thin, elastic beams, the added mass and viscous damping caused by the fluid can drastically alter the resonance frequencies and damping ratios of the cantilevers. Understanding the influence of the fluid is critical to predicting the dynamic behavior of the vibrating cantilever. This topic has been widely investigated over the past decades, and the interested reader is directed to books by Blevins (1990) and Paidoussis (2004) for a detailed treatment of flow-induced vibration and fluid-structure interaction. Applications of interest for a cantilever beam vibrating in a fluid span more than nine orders of magnitude (nanometer to meter) in length scales. Micro- and nano-scale applications of cantilevers include biosensors (Gupta et al. 2006 and Ilic et al. 2001), atomic force microscope cantilevers (Binnig 1986), and rheological measurement devices (Boskovic et al. 2002). At larger length scales, applications such as piezoelectric fans for electronics cooling (Açıalın et al. 2004) and Kimber et al. 2007) and flapping wings for propulsion (Shyy et al. 1999) involve vibrating cantilevers. Although different length scales are of interest depending on the application, the common feature is that the dynamic behavior is dependent on the interaction between the structure and the fluid. The fluid surrounding a vibrating beam increases the effective mass and damping of the structure. The length scales and vibration amplitudes encountered in a particular application determine if one of these effects (added mass or added damping) is dominant over the other, or if both play a large role.

Regardless of the scale under investigation, most prior studies have been primarily concerned with a single cantilever beam (Fu and Price 1987, Basak et al. 2006, and Sader 1998). Fewer studies have focused on the hydrodynamic interaction of multiple beams vibrating in close proximity. Cantilever arrays are encountered in applications such as the IBM millipede (Vettiger et al. 2002) and when mimicking the beating wings of insects (Lehmann et al. 2005).

Hosaka and Itao (2002) investigated the interaction between two cantilevers oriented such that the lengthwise-edges came in contact when the gap was zero, an orientation which will be referred to in this paper as "edge-to-edge." They considered small oscillatory Reynolds numbers and could then represent the continuous beam as a string of spheres, enabling an analytical approach to the fluid model. The degree to which the two beams were coupled was found to depend heavily on the beam size, gap between beams, and differences in the resonance frequencies of each beam. The level of interaction was seen to increase as each of these factors decreased. Basak and Raman (2007) explored the hydrodynamic coupling between 
microcantilevers using the same orientation as that considered by Hosaka and Itao (2002), but employed a boundary integral technique to solve the unsteady Stokes equations, enabling them to consider higher oscillatory Reynolds numbers and compute fluid velocities close to the thin microbeams. They determined the coupling as a function of separation distance, frequency, and relative amplitude and phase of two neighboring beams, and concluded that two beams can either constructively or destructively interfere, depending on their relative phase and separation distance. Guidelines were also formulated regarding the implementation of microscale cantilever arrays, depending on whether weak or strong coupling was desired.

Constructive and destructive interference between vibrating cantilevers was also observed by Ihara and Watanabe (1994). They considered an orientation where a pitch of zero meant that the flat surface of the two cantilevers would be in contact, a condition which will be referred to in this paper as "face-to-face." They investigated the flow field generated for in-phase and out-of-phase vibration at three different pitches. The cantilevers were sandwiched between two large plates in an attempt to approximate a two-dimensional flow field, and results were compared to the flow field generated by a single cantilever in the same experimental setup. It was found that the volumetric flow rate produced by vibrating cantilevers was substantially greater when operating in-phase. While the aerodynamic loading on the beams themselves was not explored, their study provides further evidence of the sensitivity of cantilever performance to the phase difference between beams.

Jeong et al. (2004) conducted a theoretical study on two identical, parallel plates with a fluid bounded between them and considered both in-phase and out-of-phase vibration. The plates were clamped along all edges and held at a fixed, constant spacing. The expected decrease in resonance frequencies due to the added mass effect from the fluid was found to be larger for out-of-phase vibration when compared to in-phase vibration. The damping was not computed.

These four studies focused on hydrodynamic interactions between multiple beams or surfaces (Hosaka and Itao 2002, Basak and Raman 2007, Ihara and Watanabe 1994, and Jeong et al. 2004) included small length-scale assumptions or small vibration amplitudes, and therefore, do not address the hydrodynamics of macro-sized flexible cantilevers oscillating at large amplitudes, which is the focus of the current study. In addition, two array orientations are considered in the current work, thereby broadening the applications of interest. The objective of the present work is to experimentally quantify the fluid loading (added mass and damping) on arrays of cantilevers vibrating face-to-face and edge-to-edge. One of the target applications for this study is in the cooling of electronic components, in which macro-sized cantilevers vibrating near a 
heated surface provide heat transfer enhancement. These devices are termed 'piezoelectric fans,' since they are actuated through the use of a piezoelectric material bonded to a flexible cantilever. These fans have generated great interest as they require only small amounts of input power and are easily adaptable to different geometries. In recent work by Kimber and Garimella (2008), constructive interference was observed in the cooling performance from an array of piezoelectric fans under certain conditions. Fig. 1(a) shows the orientation considered in that work as well as typical temperature fields produced on the heated surface under the action of the flow fields generated by fans at both a large pitch [Fig. 1(b)] and a small pitch

[Fig. 1(c)]. The greatest temperature reductions are obtained within the vibration envelopes of each fan, with the smaller of the two pitches providing the better cooling performance. A model to describe the dynamic behavior of such fan arrays is first discussed in the following, after which the experimental setup is described. Results are then presented for the aerodynamic damping observed as well as the control of the extent of this damping by controlling the pitch between neighboring beams in the array.

\section{NOMENCLATURE}

$\begin{array}{ll}A & \text { vibration amplitude } \\ A_{\max } & \text { maximum vibration amplitude (A at resonance) } \\ A_{s t} & \text { static displacement }\left(F_{0} / k\right) \\ C & \text { viscous damping coefficient } \\ D & \text { piezoelectric fan width } \\ F_{0} & \text { magnitude of driving force } \\ k & \text { spring stiffness of beam } \\ m & \text { mass } \\ P & \text { fan pitch } \\ Q & \text { quality factor } \\ t & \text { time }\end{array}$

Greek Symbols

$\omega$ driving frequency

$\omega_{n}$ resonance frequency in vacuum

$\zeta \quad$ damping ratio in vacuum

Subscripts 
a aerodynamic component

air quantity measured in air

iso isolated (uncoupled) value

1,2 fan number

\section{Modeling Dynamic Behavior}

The dynamics of a thin, flexible beam can be approximated using a single-degree-of-freedom system with equivalent stiffness $(k)$, mass $(m)$, and damping $(c)$. A sinusoidally varying external force is applied with a specified magnitude $\left(F_{0}\right)$ and frequency $(\omega)$. The displacement of the beam can then be described by the following differential equation:

$$
m \ddot{x}+c \dot{x}+k x=F_{0} \sin (\omega t)
$$

The solution to this differential equation yields the frequency response of the beam. Using traditional definitions for resonance frequency $\left(\omega_{n}=\sqrt{k / m}\right)$ and damping ratio $\left[\zeta=c /\left(2 m \omega_{n}\right)\right]$, the expression for amplitude $(A)$ takes the following form:

$$
\frac{A \cdot k}{F_{0}}=\left\{\left[1-\left(\frac{\omega}{\omega_{n}}\right)^{2}\right]^{2}+\left[2 \zeta\left(\frac{\omega}{\omega_{n}}\right)\right]^{2}\right\}^{-1 / 2}
$$

Here, $A$ is normalized by the static displacement $\left(F_{0} / k\right)$. The foregoing discussion is applicable only when

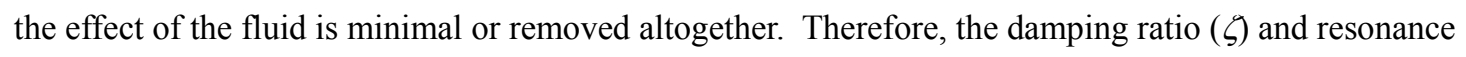
frequency $\left(\omega_{n}\right)$ in Eq. (2) represent structural quantities, or those measured in vacuum conditions. As previously discussed, the loading from the fluid is manifested as an added mass and added damping so that the equivalent mass and damping when measured in air become:

$$
m_{\text {air }}=m+m_{a} ; \quad c_{\text {air }}=c+c_{a}
$$

where $m_{a}$ and $c_{a}$ are the aerodynamic components of mass and damping, respectively. Substituting $m_{\text {air }}$ and $c_{a i r}$ in place of $m$ and $c$ in Eq. (1) and solving the new differential equation yields the following expression for amplitude with the effects of the air included:

$$
\frac{A \cdot k}{F_{0}}=\left\{\left[1-\left(1+\frac{m_{a}}{m}\right)\left(\frac{\omega}{\omega_{n}}\right)^{2}\right]^{2}+\left[2 \zeta\left(1+\frac{c_{a}}{c}\right)\left(\frac{\omega}{\omega_{n}}\right)\right]^{2}\right\}^{-1 / 2}
$$


If the influence of the fluid were negligible, then $m_{a}=c_{a}=0$, and Eq. (4) reduces to Eq. (2). The loading from the fluid on the beam causes an inclusion of mass and viscous damping in the equation. However, it is more helpful to discuss this loading from the fluid in terms of the shift in resonance frequency and increase in the damping ratio, when compared to the values obtained under vacuum conditions. The measured resonance frequency in air $\left(\omega_{n, \text { air }}\right)$ can be expressed in terms of the resonance frequency in vacuum $\left(\omega_{n}\right)$ and the ratio of aerodynamic to structural mass $\left(m_{a} / m\right)$ according to:

$$
\omega_{n, a i r}=\omega_{n}\left(1+\frac{m_{a}}{m}\right)^{-1 / 2}
$$

The damping ratio measured in air $\left(\zeta_{\text {air }}\right)$ can likewise be determined from the damping ratio in vacuum $(\zeta)$ and the ratio of aerodynamic to structural damping factors $\left(c_{a} / c\right)$ from:

$$
\zeta_{\text {air }}=\zeta\left(1+\frac{c_{a}}{c}\right)
$$

This damping ratio can also be expressed in terms of the aerodynamic damping ratio $\left(\zeta_{a}\right)$ as:

$$
\zeta_{\text {air }}=\zeta+\zeta_{a}
$$

Comparison between Eqs. (6) and (7) reveals that:

$$
\zeta_{a}=\zeta \frac{c_{a}}{c}
$$

In this work, the damping ratios will be expressed in terms of the quality factor $(Q)$ such that (Ewins, 2000):

$$
Q=1 / 2 \zeta ; \quad Q_{a i r}=1 / 2 \zeta_{\text {air }} ; \quad Q_{a}=1 / 2 \zeta_{a} ;
$$

From a damping perspective, the quantity of most interest is the aerodynamic quality factor $\left(Q_{a}\right)$, which is determined from:

$$
Q_{a}=\left(\frac{1}{Q_{a i r}}-\frac{1}{Q}\right)^{-1}
$$

It should be noted that the aerodynamic damping $\left(c_{a}\right)$ is amplitude-dependent. As a result, $\zeta_{a}$ and $\zeta_{\text {air }}$ (and thus $Q_{a}$ and $Q_{a i r}$ ) are also functions of vibration amplitude. Bidkar et al. (2009) investigated this amplitudedependence for a single beam and presented an analytical approach for predicting $Q_{a}$ in terms of the beam geometry and vibration amplitude and frequency. Their modeling approach was validated with experiments involving piezoelectric fans vibrating in vacuum and air. In contrast, the present work is focused on arrays of beams, and the behavior of greatest interest is that of $Q_{a}$ for the array relative to the experimental value of $Q_{a, i s o}$, the aerodynamic quality factor in the case where the beams are spaced far enough apart to be isolated 
and not interact with their neighbors. In addition to the driving force $\left(F_{0}\right)$, the driving frequency $(\omega)$ also influences the vibration amplitude. However, the frequency targeted in the current investigation is the fundamental resonance frequency, and therefore the range of driving frequencies considered only varies by approximately $\pm 5 \%$. Therefore the $Q_{a}$ found from a frequency sweep at a fixed $F_{0}$ is assumed to be a constant value.

Besides viscous damping, an additional component of the fluid loading is the added mass. For lightly damped systems (as is the case in the current investigation), the resonance frequency is equal to the driving frequency where vibration amplitudes are largest. Therefore, $\omega_{n}$ and $\omega_{n, \text { air }}\left(\right.$ as well as $\omega_{n, \text { air,iso }}$ ) can be estimated simply by determining the location of the peak amplitude. The ratio of aerodynamic to structural mass $\left(m_{a} / m\right)$ can then easily be found from Eq.(5), even before damping is considered. Because the system is only lightly damped, this can be done independently from any damping analysis. However for the current investigation of cantilever beams vibrating in air, it will be shown that the changes in resonance frequency are small compared to the corresponding changes in damping.

Experiments are conducted in air and vacuum to determine $\omega_{n}, Q, \omega_{n, a i r}$, and $Q_{a}$. The setups are now described for the experiments in air and vacuum, along with experimental results from each set of experiments. This is followed by a detailed analysis of the behavior of the aerodynamic quality factor $Q_{a}$ for an array, relative to its value for isolated beams $Q_{a, i s o}$, as a function of distance between beams.

\section{Experimental Setups}

Two sets of experiments are conducted in this work: 1) in air, to determine $\omega_{n, \text { air }}$, and $Q_{\text {air }}$ for a range of pitches and input voltages, and 2) within a vacuum chamber, where the effect of the air is removed so that the magnitude of $\omega_{n}$ and $\zeta$ may be determined, thereby allowing $m_{a} / m$ and $Q_{a}$ to be extracted from the results obtained in the first set of experiments in air.

\subsection{Experiments in Air}

The piezoelectric fans used to analyze aerodynamic damping and added mass are shown in Fig. 2(a), illustrating the face-to-face configuration. In this image, the exposure time of the camera is increased to show the envelopes of vibration. These fans are referred to as $\mathrm{Fan}_{1}$ and $\mathrm{Fan}_{2}$, and are commercial products made from a flexible mylar blade, upon which a PZT patch is bonded near the base, thereby providing the actuation needed for vibration. They have an overall length of approximately $75 \mathrm{~mm}$, a width of $12.7 \mathrm{~mm}$, 
and a fundamental resonance frequency near $60 \mathrm{~Hz}$. The largest vibration amplitudes experienced at the fan tip are approximately $10 \mathrm{~mm}$. The fans are mounted on a stage with two isolated mounts to precisely control the pitch between the fans. Shown in Fig. 2(b) and (c) are the in-phase and out-of-phase vibration, respectively, for the face-to-face configuration, while the corresponding edge-to-edge configurations are shown in Fig. 3(a) and (b). The pitch is defined as the distance between fans for the face-to-face configuration (see Fig. 2) and the distance between fan centers for the edge-to-edge configuration (see Fig. 3). For all experiments, two laser displacement sensors (Keyence LK-G157) are positioned to capture the vibration signal of each fan independently. The sensors operate based on triangulation, under the premise that the measurement surface is a diffuse reflector. The actual point of measurement (i.e., location where the laser beam strikes the fan surface) is $4 \mathrm{~mm}$ from the fan tips. While the two fans are nominally identical, differences in fabrication cause them to have slightly different resonance frequencies. Because of this, the output phase between the fans is carefully monitored with the lasers, and a two-channel function generator (Tektronix AFG3022) is employed to provide the input signals to each fan independently. For all experiments performed in air, a controlled phase difference between the two input signals is introduced in order to ensure that appropriate conditions are maintained at the output (whether considering in-phase or outof-phase vibration). Each of these signals is routed to separate amplifiers in order to obtain the voltages needed to operate the fans.

The experimental parameters of interest are the input voltage to the piezoelectric element and the pitch (or array spacing). The ranges considered for these variables are shown in Table 1 for both face-to-face and edge-to-edge configurations. For the input voltage, the same four values $(20,50,80$, and $100 \mathrm{~V})$ are used regardless of orientation, or whether the vibration is in-phase or out-of-phase. The pitch, on the other hand is selected depending on the orientation and phase difference under investigation. For the face-to-face orientation, it is anticipated that the pitch at which the two fans begin to show coupling interactions through the fluid will depend heavily on the vibration amplitude. Therefore, the pitch for this orientation is normalized by the maximum vibration amplitude experienced at that particular pitch. These are nominal target values, and the actual values depend on the maximum amplitude at a particular pitch as measured in the experiments. It is also noted that the minimum pitch attainable for out-of-phase vibration for the face-toface configuration is a function of the amplitude only. Assuming that the fans vibrate at the same amplitude, they must be spaced a distance of at least $2 A$ apart in order to avoid collision of the fan tips, as is apparent from Fig. 2 (c). While in-phase vibration in the same orientation does not have a similar limitation [see Fig. 
2 (b)], the smallest pitch that could be reached in the experimental setup was $4 \mathrm{~mm}$. The smallest $P / A_{\max }$ considered for in-phase vibration is approximately 0.75 . For the edge-to-edge orientation, the coupling is anticipated to be more sensitive to the fan width, $D=12.7 \mathrm{~mm}$. Therefore, the pitch is normalized by the fan width for this orientation and the same range is considered for both in-phase and out-of-phase vibration (from $P / D=1.1$ to $P / D=3.0)$.

For each experiment conducted to estimate the resonance frequency and damping ratio, the following procedure is adopted:

1) select frequency range for investigation as the bandwidth of vibration (frequencies above and below resonance where amplitude reaches $\left.0.707 \cdot A_{\max }\right)$,

2) sweep through this range in small increments (40-50 points per sweep), capturing vibration amplitude and relative phase (input to output) information at each frequency, and

3) determine static displacement $\left(F_{0} / k\right)$ using same driving voltage by applying a $1 \mathrm{~Hz}$ sine wave. The amplitude vs. frequency data are then normalized by the corresponding static displacement, and the circle-fit method (Ewins, 2000) is employed to estimate the resonance frequency and quality factor for the experiments in air $\left(\omega_{n \text {,air }}\right.$ and $\left.Q_{\text {air }}\right)$ and in vacuum $\left(\omega_{n}\right.$ and $\left.Q\right)$. This method takes the raw (unsmoothed) phase and magnitude data from each frequency sweep, converted into their real and imaginary components. This yields a circular-shaped curve when displayed on the real and imaginary axes and a least-squares curve fit of the data is performed to ultimately extract the resonance frequency and quality factor. Further details of this procedure are available in Ewins (2000).

For large vibration amplitudes, the curvature of the fan is not negligible such that the location of the tip relative to its zero position has a two-component displacement. A sensitivity analysis was conducted regarding the dependence of $\zeta$ (and thus $Q$ ) on the location along the beam at which the laser is targeted. The location of the laser was varied from $2 \mathrm{~mm}$ to $10 \mathrm{~mm}$ from the fan tip in $2 \mathrm{~mm}$ increments, yielding 5 sample points. With an input voltage of $110 \mathrm{~V}$, the five values of $\zeta$ obtained varied by only $3.0 \%$.

In addition, the tolerance adopted for the precision of the phase difference is $\pm 2^{\circ}$ (i.e., conditions maintained are $0 \pm 2^{\circ}$ and $180 \pm 2^{\circ}$ for in-phase and out-of-phase vibration, respectively). An additional analysis was performed to gauge the sensitivity of damping to the actual output phase difference. In order to test this, a new tolerance of $\pm 0.5^{\circ}$ is specified, and the output phase difference is varied from $0^{\circ}$ to $12^{\circ}$ in $3^{\circ}$ increments, with the input voltage held fixed at $110 \mathrm{~V}$. The results indicate that the damping ratio changes by 
a maximum of $2.7 \%$ from 0 to 12 degrees. However, results for a phase difference of 0 and $180 \pm 2^{\circ}$ compared to those for 0 and $180 \pm 0.5^{\circ}$ revealed no significant difference (less than $1.0 \%$ ). Therefore, the originally suggested tolerance of $\pm 2^{\circ}$ is considered sufficient.

The overall experimental uncertainty has contributions from bias errors, model errors, and precision errors. The bias errors included in the uncertainty analysis are errors due to the curvature of the fan at large amplitudes $(3.0 \%)$ and control of the phase difference between the two fans $(1.0 \%)$. The precision errors are due to the experimental equipment such as the laser displacement sensor (amplitude measurement accuracy of $0.1 \%$ ), function generator (driving frequency accuracy of $0.05 \%$ ) and the data acquisition hardware $(1.0 \%$ for the voltage range encountered during the experiments). The total precision error is dominated by the data acquisition hardware and quantified as $1.0 \%$. The model error is introduced through the linear treatment of an essentially non-linear phenomenon. It is noted that the circle-fit method itself is based on a linear assumption; therefore the model error is quantified by analyzing the quality of fit for the least-squares expression determined for the circle. Any variation of actual data from a circular shape would suggest that the damping in the range considered exhibits non-linear behavior. Errors encountered for each least-squares fit represent the total model error, and for a conservative estimate, the largest model error encountered is $0.5 \%$ and is adopted for the overall uncertainty analysis. Combining these three errors (bias of $3.0 \%$ and $1.0 \%$, precision of $1.0 \%$ and model of $0.5 \%$ ) in the sum of squares method yields an overall uncertainty of $3.4 \%$ for $Q$ or $\zeta$ measured in air or vacuum. It should be noted that the parameter of interest is the aerodynamic quality factor $\left(Q_{a}\right)$ which is dependent on two measured quantities, namely $Q_{a i r}$ and $Q$ according to Eq. (10). Therefore, the percent uncertainty in $Q_{a}$ (defined as $u_{Q a}$ ) is determined according to (Figliola and Beasley, 2006):

$$
u_{Q a}=0.034\left[\left(\frac{Q_{a}}{Q_{a i r}}\right)^{2}+\left(\frac{Q_{a}}{Q}\right)^{2}\right]^{1 / 2}
$$

This is determined independently for each value of $Q_{a}$ and ranges from $4.1 \%$ to $7.4 \%$ across all experiments.

\subsection{Experiments in Vacuum}

The experiments conducted under vacuum conditions serve two purposes. The first has been discussed earlier and is essential in order to extract $Q_{a}$ in Eq. (10) from the air experiments. In addition, these experiments also help verify that the coupling phenomenon is indeed due to fluid-structure interaction and that structural coupling can be neglected. The same two piezoelectric fans used in the air experiments are 
mounted in the face-to-face configuration in a vacuum chamber as shown in Fig. 4(a) for the vacuum experiments. This chamber is sufficiently large such that the effect of the chamber walls on the vibration of the fans is negligible, as the walls are more than 13 amplitudes away from the fans. As will be shown in section 5 this distance is more than adequate to justify this assumption. Ports are provided for electrical inputs to the fans, and an optical viewport provides the capability to capture vibration signals as shown in Fig. 4(b). A laser displacement sensor is used to obtain the displacement signal from the fan nearest to the viewport, and because vibration data is only attainable for a single fan, the phase difference between the outputs cannot be monitored for the vacuum experiments. A high vacuum pump (Edwards E2M2) is used to create near-vacuum conditions, whereby the pressure within the chamber is reduced to less than $10 \mathrm{~Pa}$. The same procedures outlined for the air experiments are also used for all vacuum experiments. It should be noted that the static displacements used in the subsequent analysis are those obtained under vacuum conditions as suggested by Eq. (2).

\section{Results}

The experimental results for fans vibrating in air are presented first to illustrate the coupling phenomenon by which two fans influence the dynamics of each other. The results obtained under vacuum conditions are then discussed to delineate the influence of air on the fans.

\subsection{Coupling Phenomena in Air}

In order to illustrate the coupling effect, the fans are mounted in the face-to-face configuration, and forced to vibrate in-phase. Amplitude versus frequency curves are shown at three different pitches in Fig. 5(a) and (b) for $\mathrm{Fan}_{1}$ and $\mathrm{Fan}_{2}$, respectively. For all data points in both these plots, the driving voltage is fixed at $80 \mathrm{~V}$. Each fan has its own unique fundamental resonance frequency, which when uncoupled from the other fan is experimentally determined to be $60.80 \mathrm{~Hz}$ and $60.15 \mathrm{~Hz}$ (not shown on the plot) for Fan and $\mathrm{Fan}_{2}$, respectively. The amplitude response clearly changes as the pitch decreases from $40 \mathrm{~mm}$ to $8.4 \mathrm{~mm}$ and finally to $5 \mathrm{~mm}$. The trend observed in these two plots shows that the vibrating fans are able to achieve higher amplitudes as the pitch is decreased. This is true for both Fan 1 and Fan ${ }_{2}$, with the maximum amplitudes being over $45 \%$ larger for the $P=5 \mathrm{~mm}$ case $\left(A_{\max } \approx 10.4 \mathrm{~mm}\right)$ compared to $P=40 \mathrm{~mm}$ case $\left(A_{\max } \approx 7 \mathrm{~mm}\right)$ 
As the relative sharpness of the resonance peak is a measure of the damping, it is readily apparent that this quantity is also highly sensitive to the pitch. The resonance peaks become sharper as the pitch is decreased, suggesting that the damping ratio is decreasing and the quality factor is increasing. The viscous dissipation in the fluid is thus seen to become smaller as the pitch is decreased. There is also a slight change in resonance frequency for the three pitches considered in Fig. 5(a) and (b). The resonance frequencies for $P$ $=5,8.4$, and $40 \mathrm{~mm}$ are respectively $60.98,60.81$, and $60.67 \mathrm{~Hz}$ for $\mathrm{Fan}_{1}$ and $60.70,60.45$, and $60.20 \mathrm{~Hz}$ for $\mathrm{Fan}_{2}$, suggesting that the added mass from the fluid becomes smaller as the pitch is decreased. The overall trends of both $\omega_{n, \text { air }}$ and $\zeta_{\text {air }}$ suggest that the effect of the fluid is lessened when the two fans vibrate in-phase and the pitch is small, as would be intuitively expected. If the effect of the air were removed altogether for these vibrating beams, the expected result would be a higher resonance frequency, and lower values of damping. That this is indeed the case will be shown with the vacuum experiments.

For the same two fans in the same orientation (face-to-face), the trends observed for out-of-phase vibration are almost exactly opposite to those seen for in-phase vibration above, as illustrated in Fig. 6(a) for data collected from $\mathrm{Fan}_{1}$. Similar results were found for Fan 2 . At a sufficiently large pitch $(P=40 \mathrm{~mm})$, the difference between in-phase and out-of-phase vibration is minimal. However as the pitch is decreased $(P=$ $14 \mathrm{~mm}$ ), the importance of phase difference becomes readily apparent. In contrast to the $12 \%$ increase in $A_{\text {max }}$ from $P=40 \mathrm{~mm}$ to $P=14 \mathrm{~mm}$ observed for in-phase vibration, an $11 \%$ decrease is observed for out-ofphase vibration for the same change in pitch. It is noted that the vibration amplitude for all four data sets presented in Fig. 6(a) becomes nearly indistinguishable when the driving frequency is $2-3 \mathrm{~Hz}$ below the resonance frequency. For a given fan pitch, the fans thus only appear to become coupled when the vibration amplitude reaches a certain level. Upon closer observation, it is also concluded from Fig. 6 that the distance at which two vibrating beams become coupled is larger for in-phase vibration than for out-of-phase vibration (i.e., fans vibrating out-of-phase will become coupled sooner). Compared to the $\phi_{180}$ curve at $P=40 \mathrm{~mm}$, the $\phi_{180}$ curve at $P=14 \mathrm{~mm}$ starts deviating at $\mathrm{A} \approx 5 \mathrm{~mm}$ (i.e., $P / A \approx 2.8$ ). For the $\phi_{0}$ curves, this occurs at $\mathrm{A}$ $\approx 6 \mathrm{~mm}$ (i.e., $P / A \approx 2.3$ ). Similar experiments performed with fans in the edge-to-edge configuration are shown in Fig. 6(b). The damping again is seen to depend heavily on the pitch, but the effect of phase is, however, opposite relative to the face-to-face configuration. The damping is seen to decrease for out-ofphase vibration and increase for in-phase vibration. The fact that out-of-phase vibration causes a reduction in damping for the edge-to-edge configuration while in-phase vibration causes the same behavior for the faceto-face configuration is explained by the fact that in either configuration, the reduction in damping occurs 
when the fans travel in the low-pressure wake of their neighbor. One of the main purposes of this paper is to quantify and predict this reduced damping from a vibration standpoint, which will be discussed in a later section.

\subsection{Behavior in Vacuum}

Experiments in the vacuum setup are used to estimate $\omega_{n}$ and $\zeta$. The additional motivation for these experiments is to verify that the observed coupling phenomena are indeed due to fluid-structure interaction, and not due to structural coupling in the test apparatus. $\mathrm{Fan}_{1}$ and $\mathrm{Fan}_{2}$ are tested individually to meet the first objective. For the second, the two fans are tested in a face-to-face configuration at a fixed pitch of $P=$ $15 \mathrm{~mm}$; the tests are conducted both in a vacuum and in air (i.e., without pulling a vacuum) in the same setup to maintain otherwise identical conditions. For these experiments, in-phase excitation, rather than in-phase vibration is employed since it was not possible to measure the vibration signal of both fans in this setup. However, as will be shown, this is sufficient in order to determine the source of coupling.

Amplitude and frequency data are shown for a single fan and for two fans in Fig. 7(a) and (b) for air and vacuum conditions, respectively. All data shown are for Fan ${ }_{1}$ with the amplitude normalized by the respective static displacement $\left(A_{s t}\right)$ in order to readily compare the sharpness of resonance peaks in vacuum and air. These experiments were conducted at driving voltages of $80 \mathrm{~V}$ and $6 \mathrm{~V}$ for air and vacuum experiments, respectively. As seen before from the experiments in air, the presence of a second fan causes the damping to decrease and the resonance peak to become sharper (Fig. 7(a)). This coupling effect is virtually non-existent in vacuum conditions as seen in Fig. 7(b), where the amplitude response is independent of whether one or two fans are considered. There are also differences between the single fan curve in air (Fig. 7(a)) and in vacuum (Fig. 7(b)). As expected, the resonance frequency is lower when air is present (60.8 Hz in air and $62.04 \mathrm{~Hz}$ in vacuum) due to the added mass from the fluid. The ratio of the aerodynamic to structural mass $\left(m_{a} / m\right)$ can be computed according to Eq. (5). As the shift in resonance frequency is small $(1.24 \mathrm{~Hz})$, the mass ratio is also small, and for this case, is equal to 0.01 . In other words, the added mass due to the fluid is $1 \%$ of the effective structural mass in vacuum.

For the cantilevers under investigation in this work, the changes in resonance frequency are quite small when comparing vacuum and air conditions; further changes in resonance frequency when comparing a single fan to two fans are even smaller. On the other hand, the changes in damping can be quite dramatic. As an example, the frequency response curve for a single fan in Fig. 7 (a) is compared to the single-fan curve in 
Fig. 7 (b). This corresponds to $\zeta_{\text {air }}=0.0194\left(Q_{\text {air }}=25.8\right)$ and $\zeta=0.00402(Q=124.5)$ for air and vacuum experiments, respectively. The aerodynamic damping can be extracted according to Eq. (7), and for this case yields $\zeta_{a}=0.0154\left(Q_{a}=32.5\right)$. The ratio of aerodynamic-to-structural damping coefficients $\left(c_{a} / c_{s}\right)$ can be determined from Eq. (8) yielding $c_{a} / c_{s}=3.83$. Therefore, for a single fan, the transition from vacuum to air which yields only a $1 \%$ increase in added mass, results in almost $400 \%$ increase in added damping. For this reason, $\zeta_{a}$ is of primary importance in understanding the aerodynamic interactions in fan arrays. Therefore, only the aerodynamic quality factor $\left(Q_{a}\right)$, which is a measure of the damping, is considered for the detailed analysis in the following section.

\section{Quality Factor Characterization}

The aerodynamic quality factor $\left(Q_{a}\right)$ is determined according to Eq. (10) for all experiments and the results are shown in Fig. 8 for Fan 1 in the face-to-face configuration. This is plotted as a function of pitch in Fig. 8(a), and dimensionless pitch $\left(P / A_{\max }\right)$ in Fig. $8(\mathrm{~b})$. Both in-phase and out-of-phase data are shown, and all of the curves are seen to approach an isolated (uncoupled) value, $Q_{a, \text { iso }}$, for each unique driving voltage. The fact that $Q_{a, i s o}$ is dependent on the driving voltage is expected as this quantity is very much dependent on the vibration amplitude, with higher levels of damping being encountered for larger vibration amplitudes (Bidkar et al., 2009). From these results, $Q_{a}$ is seen to increase as the pitch is decreased from the isolated fan value $\left(Q_{a, i s o}\right)$ for the $\phi_{0}$ curves; conversely, $Q_{a}$ decreases as the pitch is decreased for the $\phi_{180}$ curves. The rate at which this increase or decrease occurs is more readily apparent when the pitch is normalized by the maximum amplitude achieved for any particular frequency sweep, as shown in Fig. 8(b). For the $\phi_{180}$ experiments, the minimum attainable pitch is slightly larger than two times the amplitude. It is interesting to note that the four $\phi_{180}$ curves appear to be nearly parallel to each other, suggesting that a simple relationship can be deduced to capture this effect. The same is observed for the four $\phi_{0}$ curves.

Taking each curve in Fig. 8(b) and normalizing $Q_{a}$ by its isolated value, $Q_{a, \text { iso }}$, causes all the $\phi_{0}$ data to collapse to a single curve, and all the $\phi_{180}$ data to collapse on to a separate curve. The result is shown in Fig. 9(a), which includes data from both fans as well as curve fits of the form:

$$
\frac{Q_{a}}{Q_{a, \text { iso }}}=C_{1}\left(\frac{P}{A_{\max }}\right)^{n}+1
$$


where the exponent, $n$, is a negative number so that as the pitch becomes large, the ratio on the left hand side of Eq. (12) approaches unity, meaning that $Q_{a}$ converges to $Q_{a, \text { iso. }}$. The coefficient $C_{1}$ and exponent $n$ are found for each expression using a least-squares analysis of the data, yielding $C_{1}=0.36$ and $n=-0.99$ for $\phi_{0}$ and $C_{1}=-0.92$ and $n=-2.11$ for $\phi_{180}$. It is interesting to compare the magnitude of $n$ for $\phi_{0}(n=-0.99)$ vs. $\phi_{180}(n=-2.11)$. This suggests that the rate at which $Q_{a}$ decreases for out-of-phase vibration is much higher than the rate at which $Q_{a}$ increases for in-phase vibration. In either case, for $P / A_{\max } \approx 6$, the fans become nearly isolated with only a minimal coupling effect.

Similar analysis of the edge-to-edge configuration showed similar characteristics in that the data are easily reduced using the previously defined dimensionless pitch. This entails replacing the quantity $P / A_{\max }$ from Eq. (12) with $P / D$, yielding:

$$
\frac{Q_{a}}{Q_{a, \text { iso }}}=C_{1}\left(\frac{P}{D}\right)^{n}+1
$$

The result is shown in Fig. 9(b) along with the curve fits, with $C_{1}=-0.15$ and $n=-1.97$ for $\phi_{0}$, and $C_{1}=0.19$ and $n=-2.66$ for $\phi_{180}$. Uncoupled behavior for this orientation is attained when $P / D \approx 3$, or when the distance between the beams is approximately equal to twice their width. The relative change in $Q_{a}$ as shown in Fig. 9(b) for the edge-to-edge configuration (increase of $20 \%$ for out-of-phase and decrease of $15 \%$ for inphase vibration) is not as large as that seen for the face-to-face configuration as illustrated in Fig. 9(a) (increase of $70 \%$ for in-phase and decrease of $20 \%$ for out-of-phase vibration). This is attributed to the faceto-face orientation being such that the fans are located directly in the wake of their neighbors, while this is not the case for the edge-to-edge configuration. This suggests that the fluid surrounding a vibrating cantilever is agitated to a greater extent in locations perpendicular to the width dimension, just above and below the beam. However, the fact that coupled behavior is observed for the fans in the edge-to-edge configuration as well, suggests that locations on either side of the beam also experience a considerable level of fluid agitation.

The significance of this analysis lies in the ability to describe the damping for all experiments conducted in this work with expressions of a very simple mathematical form. For beams of geometries and resonance frequencies that are different from those considered in this work, the coefficient and exponent in Eq. (12) would likely take different values; however, a similar data reduction scheme is expected to hold. Future work could include attempts to describe the coupling from a more fundamental point of view as a function of beam material, vibration frequency, and other variable parameters. The results presented in this 
work, however, show that a high degree of coupling occurs in arrays of cantilever beams, and this can be exploited depending on whether the application targets energy dissipation or maximization of the vibration amplitude for the smallest amount of input power.

\section{Conclusions}

This paper presents an experimental effort to describe the complex aerodynamic coupling in arrays of macro-sized vibrating cantilevers. In-phase and out-of-phase vibration are considered for face-to-face and

edge-to-edge orientations. For the former orientation, a substantial decrease in the fluid damping is observed with in-phase vibration, while the converse is true with out-of-phase vibration. For the edge-to-edge configuration, out-of-phase vibration exhibits a decrease in fluid damping and in-phase vibration causes an increase. For either orientation and regardless of phase difference, the degree to which the fans become coupled increases as the pitch becomes smaller. This dependence on pitch is found to show a strong correlation to vibration amplitude for the face-to-face configuration and fan width for the edge-to-edge configuration. The effect on the dynamics of cantilever arrays is largely explained as a decrease or increase in viscous damping with changes in resonance frequency being comparatively quite small. The analysis of the data includes correlations which describe the aerodynamic quality factor of vibrating cantilever arrays as a function of vibration amplitude and pitch between neighboring components of the array. The vibrating beams are found to behave in an isolated, or uncoupled, manner when the pitch is approximately six times the amplitude for a face-to-face configuration, and approximately three times the fan width for edge-to-edge configuration.

It is emphasized that the results presented in this paper are based on beams of roughly the same resonance frequency and geometry. Additional investigations which consider fans of different materials and frequencies should be pursued in order to incorporate the beam material properties into the analysis and understand this phenomenon from a more generalized perspective. Also, to aid in actual device implementation, further analysis of the fluid damping as a function of proximity to side walls and, in the case of electronics cooling, proximity to the heated surface, are warranted.

\section{Acknowledgements}

The authors acknowledge the financial support from members of the Cooling Technologies Research Center, a National Science Foundation Industry/University Cooperative Research Center at Purdue 
University. The authors are grateful to Professor Arvind Raman, Professor Anil Bajaj, and Rahul Bidkar for their helpful discussions and insight. 


\section{References}

Açıkalın, T., Wait, S.M., Garimella, S.V., and Raman, A., 2004. Experimental Investigation of the Thermal Performance of Piezoelectric Fans. Heat Transfer Engineering 25, 4-14.

Basak, S., Raman, A., 2007. Hydrodynamic Coupling between Micromechanical Beams Oscillating in Viscous Fluids. Physics of Fluids 19, 017105.

Basak, S., Raman, A., Garimella, S.V., 2006. Hydrodynamic Loading of Microcantilevers Vibrating in Viscous Fluids. Journal of Applied Physics 99, 114906.

Bidkar, R.A., Kimber, M., Raman, A., Bajaj, A.K., Garimella, S.V., 2009. Nonlinear Aerodynamic Damping of Sharp-edged Beams at Low Keulegan-Carpenter Numbers. Journal of Fluid Mechanics (in press).

Binnig, G., Quate, C.F., Gerber, C., 1986. Atomic Force Microscope. Physical Review Letters 56, 930-933.

Blevins, R.D., 1990. Flow-Induced Vibration, 2nd Edition, Van Nostrand Reinhold, New York, NY.

Boskovic S., Chon, J.W.M, Mulvaney, P., Sader J.E., 2002. Rheological Measurements Using

Microcantilevers. Journal of Rheology 46, 891-899.

Ewins, D.J., 2000. Modal Testing: Theory, Practice and Application, 2nd Edition, Research Studies Press, Hertfordshire, England.

Figliola, R.S., Beasley, D.E., 2006. Theory and Design for Mechanical Measurements, 4th Edition, John Wiley and Sons, Hoboken, NJ.

Fu, Y., Price, W.G., 1987. Interactions between a partially or totally immersed vibrating cantilever plate and the surrounding fluid. Journal of Sound and Vibration 118, 495-513.

Gupta, A.K., Nair, P.R., Akin, D., Ladisch, M.R., Broyles, S., Alam, M.A., Bashir, R., 2006. Anomalous Resonance in a Nanomechanical Biosensor. Proceedings of the National Academy of Sciences of the United States of America 103, 13362-13367.

Hosaka, H., Itao, K., 2002. Coupled Vibration of Microcantilever Array Induced by Airflow Force. ASME Journal of Vibration and Acoustics 124, 26-32.

Ihara, A., and Watanabe, H., 1994. On the Flow Around Flexible Plates, Oscillating With Large Amplitude. Journal of Fluids and Structures 8, 601-619.

Ilic, B., Czaplewski, D., Zalalutdinov, M., Craighead, H.G., Neuzil, P., Campagnolo, C., Batt, C., 2001. Single Cell Detection with Micromechanical Oscillators. Journal of Vacuum Science and Technology B 19, 2825-2828. 
Jeong, J.H., Yoo, G.H., Lee, S.C., 2004. Hydroelastic Vibration of Two Identical Rectangular Plates. Journal of Sound and Vibration 272, 539-555.

Kimber, M., Garimella, S.V., Raman, A., 2007. Local Heat Transfer Coefficients Induced by

Piezoelectrically Actuated Vibrating Cantilevers. ASME Journal of Heat Transfer, 129, 1168-1176.

Kimber, M., Garimella, S.V., 2009. Cooling Performance of Arrays of Vibrating Cantilevers. ASME Journal of Heat Transfer (in press).

Lehmann, F.O., Sane, S.P., Dickinson, M., 2005. The Aerodynamic Effects of Wing-Wing Interaction in Flapping Insect Wings. Journal of Experimental Biology 208, 3075-3092.

Paidoussis, M.P., 2004. Fluid-Structure Interactions: Slender Structures and Axial Flow, Volume 2, Elsevier Academic Press, London, England.

Sader, J.E., 1998. Frequency Response of Cantilever Beams Immersed in Viscous Fluids with Applications to the Atomic Force Microscope. Journal of Applied Physics 84, 64-76.

Shyy, W., Berg, M., Ljungqvist, 1999. Flapping and Flexible Wings for Biological and Micro Air Vehicles. Progress in Aerospace Sciences 35, 455-505.

Vettiger, P., Cross, G., Despont, M., Drechsler, U., Durig, U., Gotsmann, B., Haberle, W., Lantz, M.A., Rothuizen, H.E., Stutz, R., Binnig, G.K., 2002. The "Millipede" - Nanotechnology Entering Data Storage. IEEE Transactions on Nanotechnology 1, 39-55. 
Table 1. List of experimental parameters considered for array experiments in air.

\begin{tabular}{lcc}
\hline Parameter & Units & Values tested \\
\hline Input voltage & $\mathrm{V}$ & $20,50,80,110$ \\
Face-to-face configuration & & \\
$\begin{array}{l}\text { Nominal dimensionless pitch } \\
\left(P / A_{\text {max }}\right), \text { in-phase vibration }\end{array}$ & $\mathrm{N} / \mathrm{A}$ & $0.75-6.0$ (7 values $)$ \\
$\begin{array}{l}\text { Nominal dimensionless pitch } \\
\left(P / A_{\text {max }}\right), \text { out-of-phase } \\
\text { vibration }\end{array}$ & $\mathrm{N} / \mathrm{A}$ & $2.1-6.0(6$ values $)$ \\
$\begin{array}{l}\text { Edge-to-edge configuration } \\
\begin{array}{l}\text { Nominal dimensionless pitch } \\
(P / D)\end{array}\end{array}$ & N/A & $1.1-3.0(7$ values $)$ \\
\hline
\end{tabular}




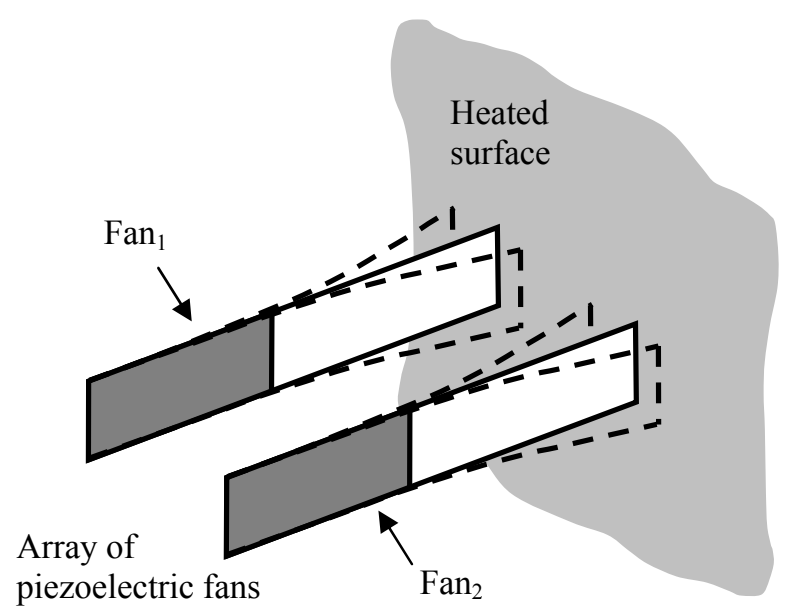

(a)
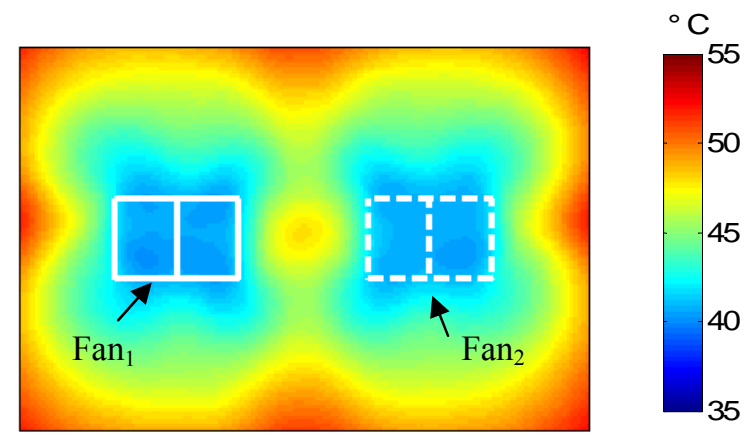

(b)


(c)

Fig. 1. Application for arrays of vibrating cantilevers: (a) illustration of piezoelectric fans used to enhance cooling of a heated surface and the resulting thermal images when (b) pitch is large and little interaction occurs between fans and (c) pitch is small with considerable amount of interaction. Temperatures are lowest near the vibrating tips of the fans. A detailed thermal analysis is available in Kimber et al. (2007) and Kimber and Garimella (2008). The lines superimposed on (a) and (b) illustrate the extent of the vibration envelope. 


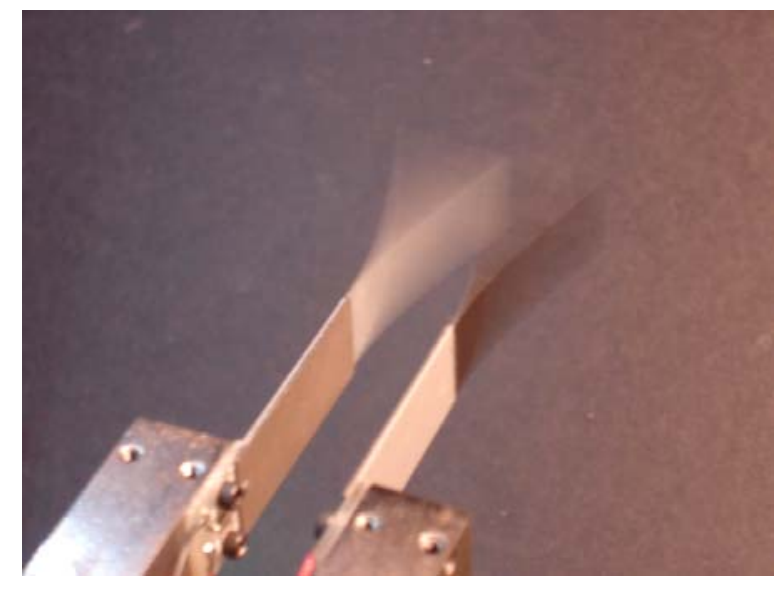

(a)

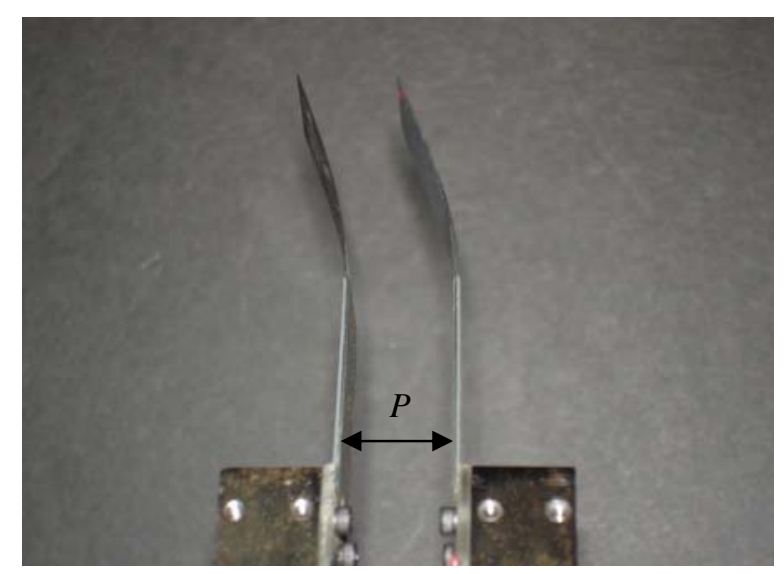

(b)

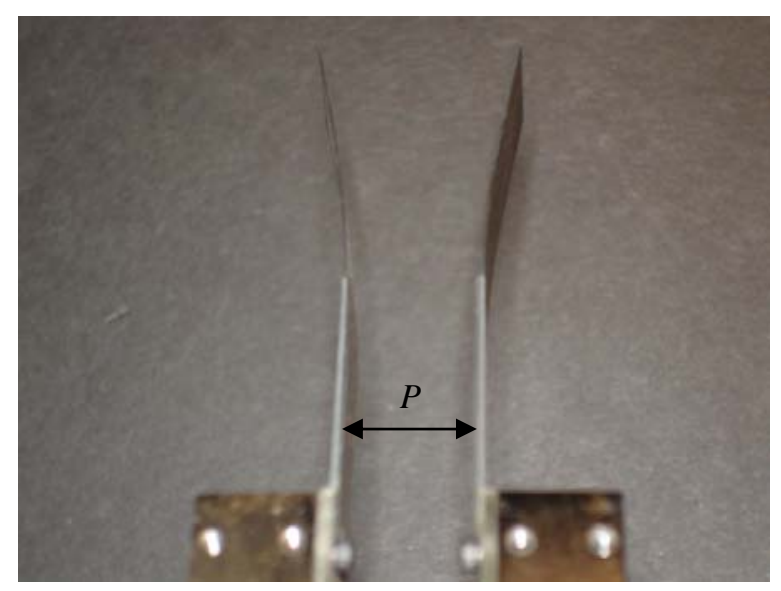

(c)

Fig. 2. Piezoelectric fans used to analyze aerodynamic damping in arrays of cantilevers in a face-to-face configuration: (a) photo of fans mounted and vibrating, (b) in-phase vibration, and (c) out-of-phase vibration. Also identified is the array pitch $(P)$. 


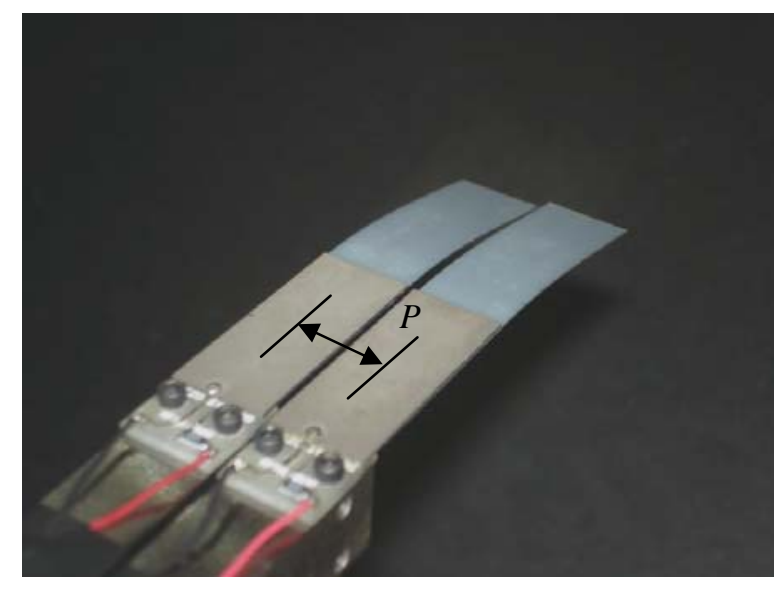

(a)

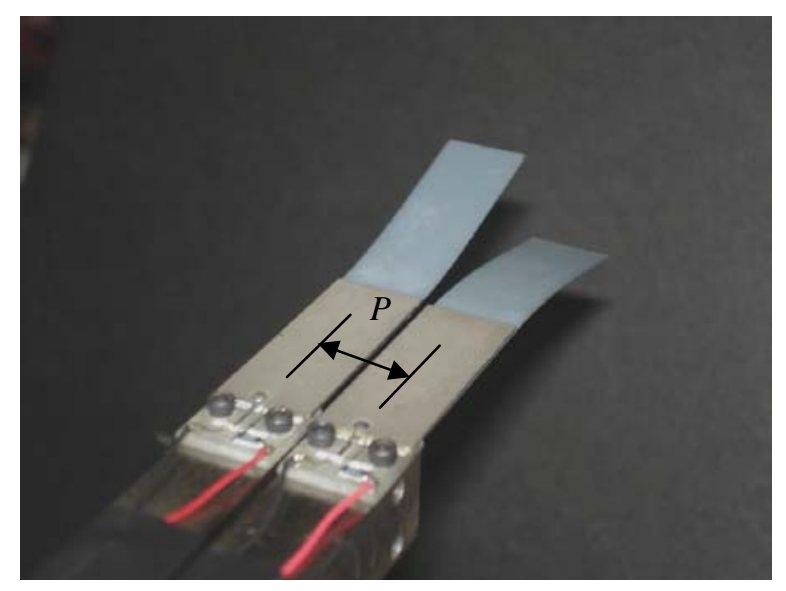

(b)

Fig. 3. Piezoelectric fans used to analyze aerodynamic damping in arrays of cantilevers in an edge-to-edge configuration: (a) in-phase vibration, and (b) out-of-phase vibration 


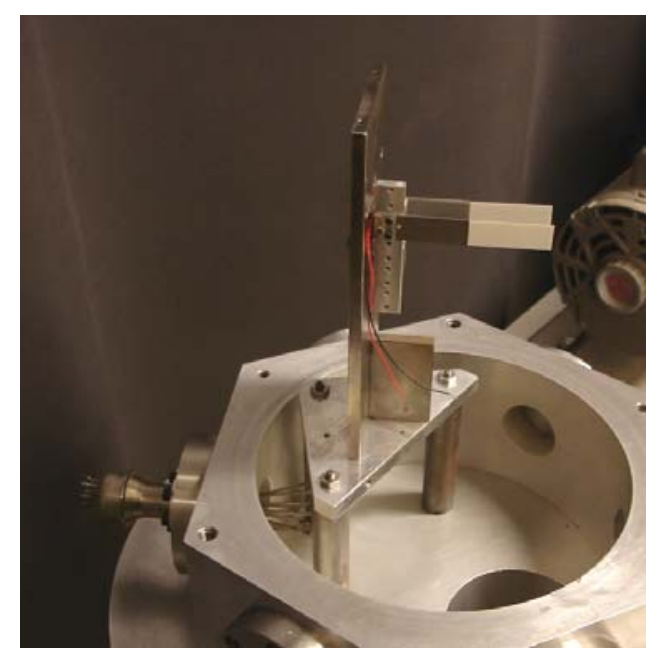

(a)

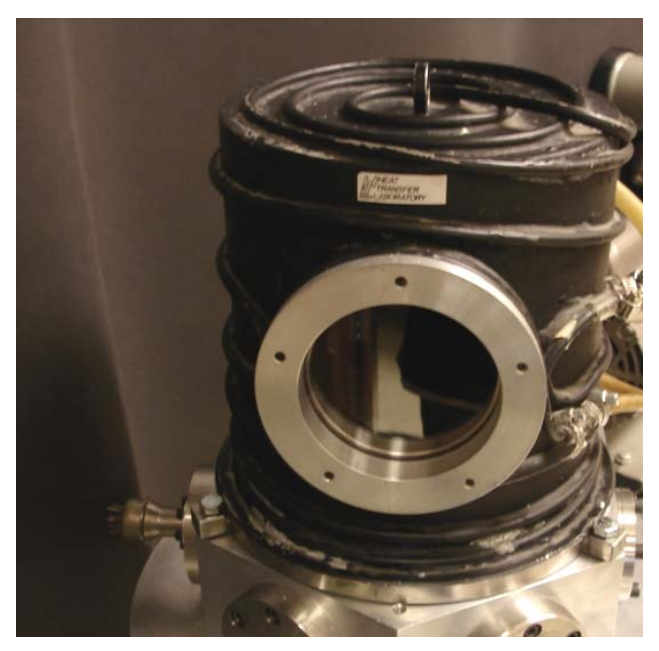

(b)

Fig. 4. Setup for vacuum chamber experiments: (a) two piezoelectric fans mounted inside in a face-to-face configuration, and (b) bell jar placed over fans with viewport for optical access. 


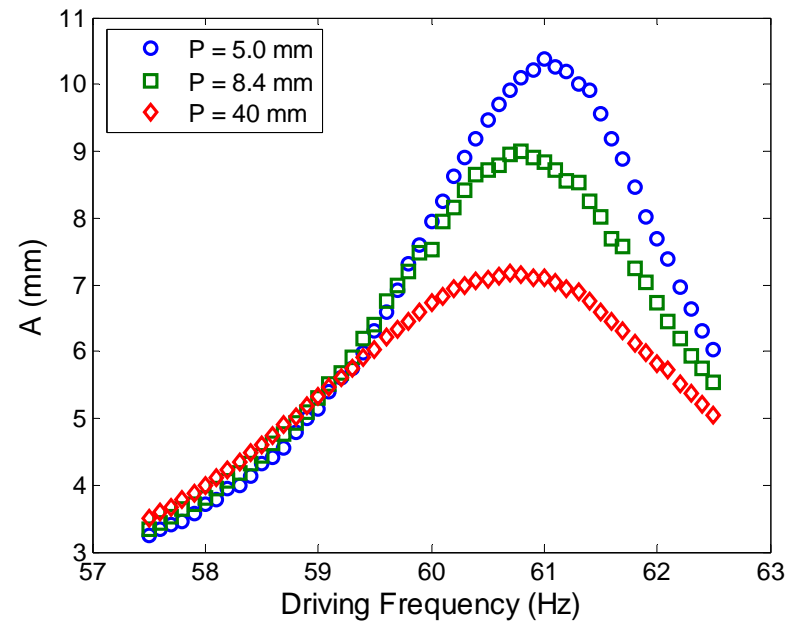

(a)

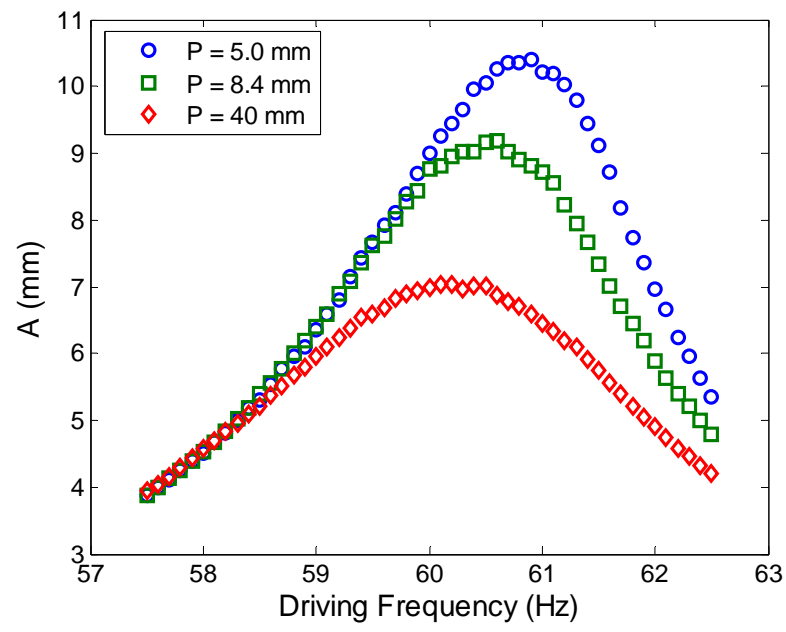

(b)

Fig. 5. Variation of amplitude with a sweep of the driving frequency in the neighborhood of the resonance frequency for (a) $\mathrm{Fan}_{1}$, and (b) $\mathrm{Fan}_{2}$. Data shown are for in-phase vibration in the face-to-face configuration with a driving voltage of $80 \mathrm{~V}$. 


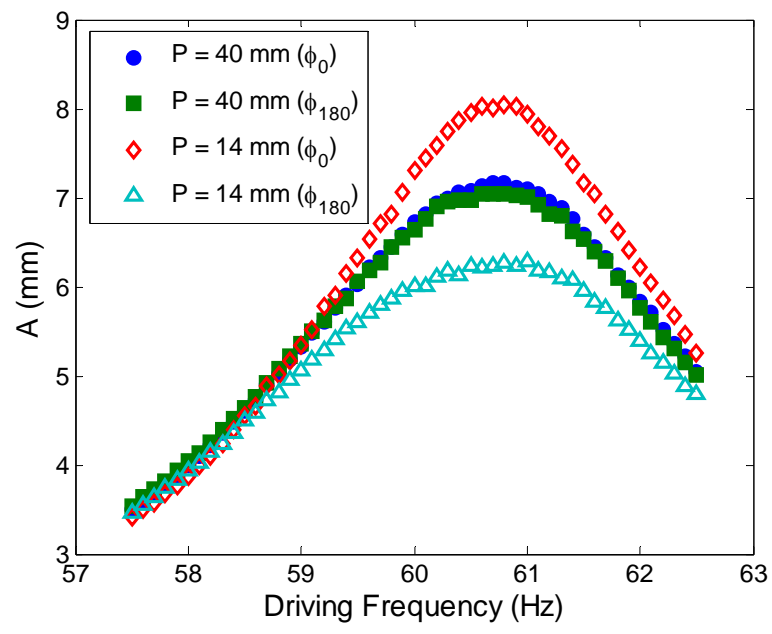

(a)

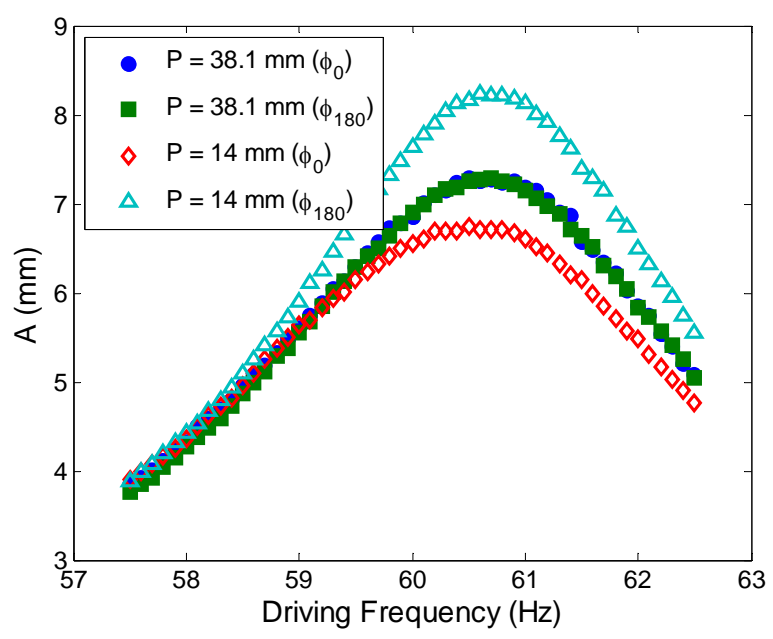

(b)

Fig. 6. Variation of amplitude with a sweep of the driving frequency in the neighborhood of the resonance frequency for $F n_{1}$ with in-phase $\left(\phi_{0}\right)$ and out-of-phase ( $\left.\phi_{180}\right)$ vibration at a large and a small pitch: (a) faceto-face configuration, and (b) edge-to-edge configuration. The driving voltage for all data shown is $80 \mathrm{~V}$. 


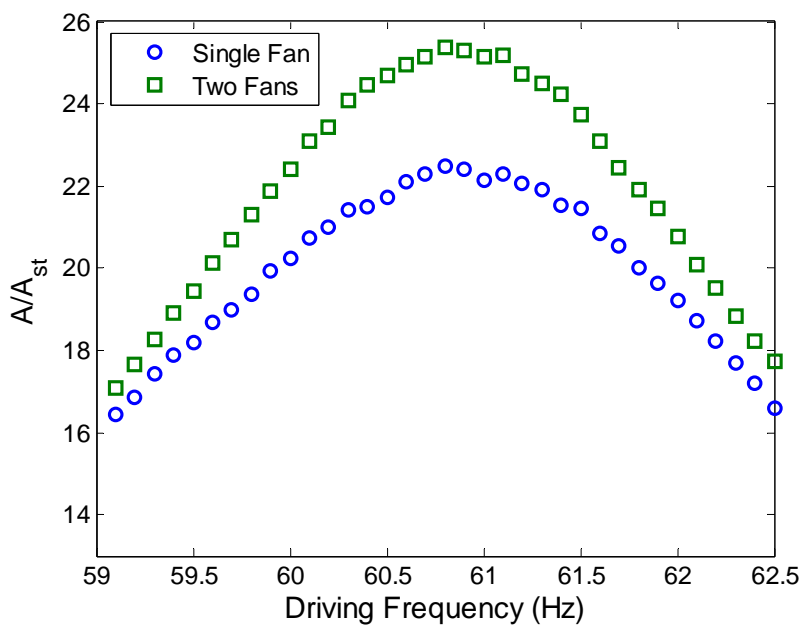

(a)

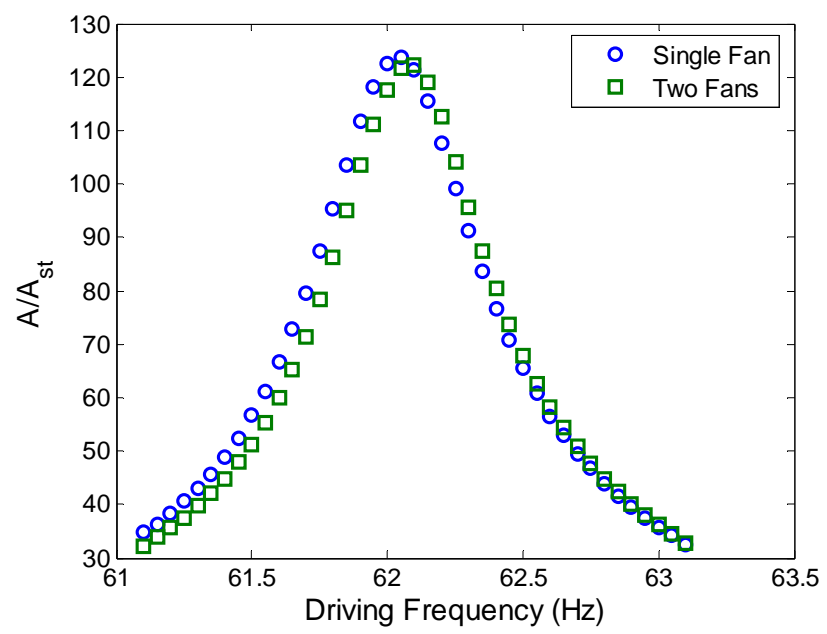

(b)

Fig. 7. Frequency and amplitude data from (a) air, and (b) vacuum experiments. The amplitude is normalized by its corresponding static displacement to illustrate the difference in damping for single- and two-fan vibration, the latter of which employs the face-to-face configuration. The data are captured in all cases from $\mathrm{Fan}_{1}$ at a driving voltage of $80 \mathrm{~V}$ in air and $6 \mathrm{~V}$ in vacuum. 




(a)



(b)

Fig. 8. Experimental estimation of quality factors for the face-to-face configuration for $\mathrm{Fan}_{1}$ as a function of (a) pitch, and (b) normalized pitch, where $A_{\max }$ is the amplitude at resonance for any given experiment. 


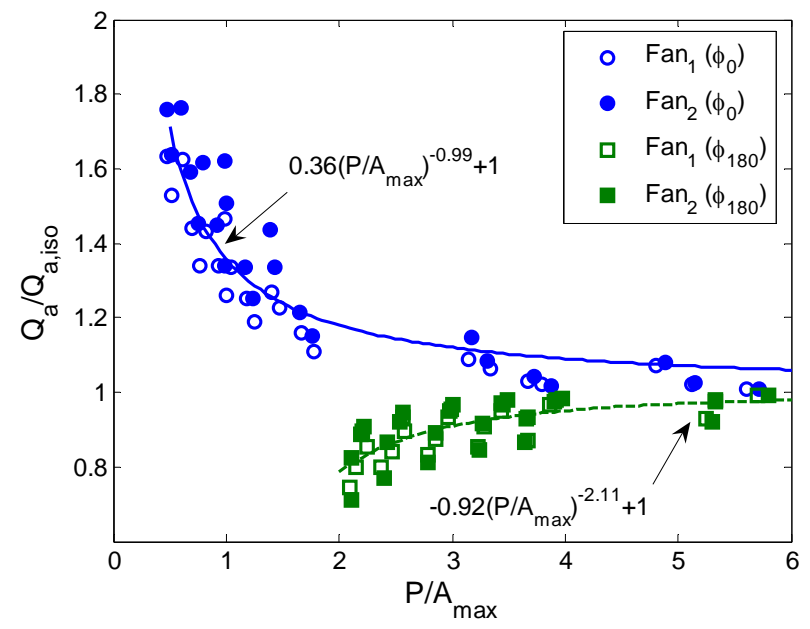

(a)

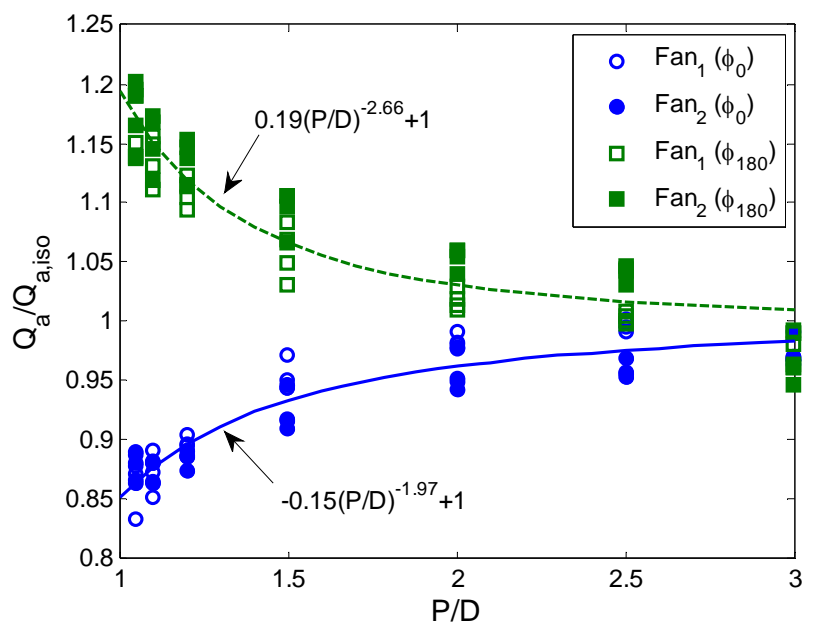

(b)

Fig. 9. Coupled quality factors normalized by their respective isolated-fan (uncoupled) values: (a) face-toface configuration, and (b) edge-to-edge configuration. Curve fits are shown for in-phase $\left(\phi_{0}\right)$ and out-ofphase $\left(\phi_{180}\right)$ vibration. 